\title{
Thermodynamic model of Ca(II)-alginate beads drying by spectrophotometry
}

\author{
Santagapita, P.R. ${ }^{\text {a,b }}$; Castro-Giraldez, M.c; Traffano-Schiffo, M.V.c; Fito, P.J. ${ }^{\text {c* }}$ \\ ${ }^{\text {a }}$ Universidad de Buenos Aires. Facultad de Ciencias Exactas y Naturales. Departamentos de Industrias \\ y Química Orgánica. Buenos Aires, Argentina

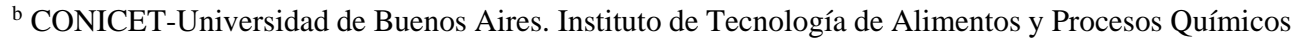 \\ (ITAPROQ). Buenos Aires, Argentina. \\ c Instituto Universitario de Ingeniería de Alimentos para el Desarrollo, Universitat Politècnica de \\ València, Camino de Vera s/n, 46022 Valencia, Spain.
}

*E-mail of the corresponding author: pedfisu@tal.upv.es

\begin{abstract}
The purpose of this work was to study the drying process of Ca(II)-alginate beads with/without sucrose, monitored by infrared thermography (IRT), in order to describe the critical points and the transformations suffered by the systems. Beads were obtained by the drop method and the drying process was performed at $40^{\circ} \mathrm{C}$ and $1.5 \mathrm{~m} / \mathrm{s}$. IRT was measured by a thermal imager Optris $P I^{\circledR} 160$. Mass, $a_{w}, x_{w}$ and volume were also obtained. It was demonstrated that IRT is a good monitoring tool, able to analyzed heat transfer in colloidal systems. A thermodynamic model able to predict the mechanical phenomena of the beads throughout drying process has been developed.
\end{abstract}

Keywords: encapsulation; thermodynamic; spectrophotometry; drying; beads. 


\section{Introduction}

Over the years, crosslinked hydrogels have been widely studied as suitable matrices for the stabilization and controlled release of biomolecules, both in the food, chemical and pharmaceutical industries ${ }^{[1]}$. Ca(II)-alginate is one of the anionic polyelectrolyte most used for the encapsulation of bioactive compounds due to its low-cost, its non-toxic character, ecofriendly and biocompatibility ${ }^{[2,3]}$. Nevertheless, $\mathrm{Ca}$ (II)-alginate shows some disadvantages such as high biomolecule leakage, low mechanical strength and large pore size ${ }^{[4]}$. Moreover, their high water activity also make them susceptible of microorganism deterioration ${ }^{[5]}$. Therefore, the coupled application of conservation treatments, such as hot air drying and the addition of protective disaccharides (as sucrose) can improve the stability of the hydrogels.

Drying operation is one of the most widely used unit operations to preserve products over time, and it involves a lot of mechanisms coupled together ${ }^{[6,7]}$. According to the traditional kinetic theory, it is divided into three main stages: induction period, constant drying velocity period and falling drying rate period ${ }^{[8]}$. An innovative technique used to control this process is the infrared thermography (IRT). IRT is an excellent method, used for studying heat transfer, which converts the radiation emitted by a body surface into temperature data without establishing contact with the object ${ }^{[9]}$. Taking into account that drying process involves a lot of mechanisms coupled together, where heat and mass transfers and mechanical effects take place simultaneously ${ }^{[10]}$, the combined used of irreversible thermodynamics and infrared thermography represents a good and promising tool.

The aim of this research is to determine the real critical points in the transformation of Ca(II)alginate beads with/without sucrose during the drying process, by means of an irreversible thermodynamic model which considers gradients of activity, temperature and mechanical energies.

\section{Materials and Methods}

\subsection{Materials}

Sodium alginate (Algogel 5540) from Cargill S.A. (San Isidro, Buenos Aires, Argentina), molecular weight of $1.97 \cdot 10^{5} \mathrm{~g} / \mathrm{mol}$ and mannuronate/guluronate ratio of 0.6 ; D-sucrose (Scharlab S.L., Barcelona, Spain), molecular weight of 342.2 g/mol. 0.05 M sodium acetate buffer pH 3.8 was prepared from acetic acid and sodium acetate (Scharlab S.L., Barcelona, Spain) ${ }^{[11]}$.

\subsection{Hydrogel beads preparation}

Two different formulations were used: alginate (A) and alginate-sucrose (AS). All the solutions were prepared in $0.05 \mathrm{M}$ sodium acetate buffer $\mathrm{pH}$ 3.8. For A beads preparation, $25 \mathrm{~mL}$ of $1 \%(\mathrm{w} / \mathrm{v})$ sodium alginate solution was dropped into $250 \mathrm{~mL}$ of $2.5 \%$ (w/v) $\mathrm{CaCl} 2$ (Scharlab S.L., Barcelona, Spain) solution. For AS preparation, a 1\% (w/v) alginate with 20\% 
(w/v) sucrose was dropped into the $2.5 \%(\mathrm{w} / \mathrm{v}) \mathrm{CaCl}_{2}$ solution supplemented with $20 \%$ (w/v) sucrose using the same procedure previously described. A peristaltic pump (Damova S.L., Barcelona, Spain, model CPM-045B) was used to $\mathrm{Ca}(\mathrm{II})$-alginate bead production according to the drop method described by Traffano-Schiffo et al., (2017), with a pump speed at $20 \pm 0$ rpm, regulated by an inverter Panasonic DV-700 (Oasaka, Japan) ${ }^{[12]}$. The $\mathrm{CaCl}_{2}$ solution (with or without sucrose) was maintained in a cold bath with constant stirring by using a vortex IKA $^{\circledR}$ MS3 basic (IKA, Staufen, Germany). A needle with $0.25 \mathrm{~mm}$ diameter and 6 mm length (Novofine 32 G, Novo Nordisk A/S, Bagsvaerd, Denmark) was used for the dropping. The distance between the needle and the $\mathrm{CaCl}_{2}$ solution was $6.0 \mathrm{~cm}$. After beads generation, they were maintained for $15 \mathrm{~min}$ in $\mathrm{CaCl}_{2}$ solution (with constant stirring), and then they were washed 5 times with bidistilled cold water $\left(5 \pm 1^{\circ} \mathrm{C}\right)$ in order to remove free $\mathrm{Ca}^{2+}$. Then, they were maintained at $4{ }^{\circ} \mathrm{C}$ until the drying treatment and further characterization.

\subsection{Drying process}

$\mathrm{Ca}(\mathrm{II})$-alginate beads were placed into the interior of a conventional air dryer. Considering previous results, the temperature and the optimal air velocity values were determined at 40 ${ }^{\circ} \mathrm{C}$ and $1.5 \mathrm{~m} / \mathrm{s}$, during a total drying time previously optimized at 22 and $24 \mathrm{~min}$ for $\mathrm{A}$ and AS, respectively. The drying air flow was kept turbulent to prevent the boundary layer from preventing inhomogeneous drying of the samples. The mass of the beads was measured continuously by a balance Mettler Toledo AB304-S (Greifensee, Switzerland) with a precision of \pm 0.001 . The infrared analysis was carried out following the method decribed by Traffano-Schiffo et al. (2014) ${ }^{[13]}$, using an infrared camera (Optris $\mathrm{PI}^{\circledR} 160$ thermal imager, Optris GmbH, Berlin, Germany) installed in front of the sample, at an angle of $45^{\circ}$ relative to the plane in which the samples were placed. The camera uses a two-dimensional Focal Plane array with $160 \times 120$ pixels, a spectral range of $7.5-13 \mu \mathrm{m}$, resolution of $0.05{ }^{\circ} \mathrm{C}$ and an accuracy of $\pm 2 \%$. A reference material of known emissivity ( $\varepsilon=0.95$ - Optris $\mathrm{GmbH}$ ) was placed next to the samples and recorded with the infrared camera with the aim to correct the emissivity of the sample. Also, the temperature of the drying air, beads, reference material and environment were measured with K-thermocouples connected to an Agilent multiplexer 34901A (Agilent Technologies, Malaysia) and registered by an Agilent Data Acquisition equipment 34972A (Agilent Technologies, Malaysia). Thermal images were analyzed by the software Optris PI Connect (Optris GmbH). After the drying treatment, the samples were kept in aqualab ${ }^{\circledR}$ disposable sample cups, sealed with parafilm ${ }^{\circledR}$ for further analysis. Mass, water activity, moisture, size and shape were measured in wet samples, immediately after drying (non-equilibrated samples) and also after $24 \mathrm{~h}$ at $4{ }^{\circ} \mathrm{C}$ in order to allow them to reach the equilibrium (equilibrated samples). 


\subsection{Hydrogel beads characterization}

Water content $\left(\mathrm{x}_{\mathrm{w}}\right)$ was obtained gravimetrically by the weight difference of the beads. The drying conditions were preosusly described by Santagapita et al. (2008). Water activity $\left(a_{w}\right)$ of beads was determined by a dew point Hygrometer Decagon (Aqualab ${ }^{\circledR}$, series $3 \mathrm{TE}$, Decagon Devices, Pullman, WA, USA), using a special sample holder. A calibration curve was performed with saturated salt solutions of known $\mathrm{a}_{\mathrm{w}}$. Measurements were made in duplicate. The size and shape were analyzed through digital images captured by a digital camera and analyzed by the free license software ImageJ (http://rsbweb.nih.gov/ij/), as was described by Aguirre Calvo \& Santagapita (2016) ${ }^{[14]}$. At least 30 wet or dried beads of each system were analyzed.

\section{Results and discussion}

In order to obtain the surface (interfacial) temperature during beads drying, an infrared model has been developed. There are two phenomena involved in the transformation of the energy that reaches the pyrosensor of the infrared camera at the real surface temperature of the sample. Firstly, the fluid located between the pyrosensor and the emitter sample (in this case is air) could be not totally transmitter, inducing a default error to the energy received. Secondly, the energy of the environment produces an overestimation of the energy emitted by the surface of the sample, adding an error in excess for the measured temperature. Therefore, it is necessary to include these phenomena as was previously developed by Traffano-Schiffo et al. (2014) [13].

In order to calculate the real temperature that is emitted by the surface of the sample obtained by the infrared camera, it is necessary to know the emissivity of the sample. Thus, comparing the temperature of the reference surface obtained by the thermocouple and the infrared camera (corrected with its known emissivity $\varepsilon_{\text {ref }}=0.95$ ), it is possible to calculate the energy being overestimated by reflection of the environment (Eq. 1). Once this energy is known, with the temperature data obtained with the thermocouple and the camera, it is possible to calculate the emissivity of the sample.

$$
E_{T}^{r e f}=\varepsilon_{r e f} \cdot \sigma \cdot T_{r e f}^{4}+E_{\text {surr }}
$$

Where $E_{T}^{\text {ref }}$ is the energy flux of the reference material $\left(\mathrm{W} / \mathrm{m}^{2}\right)$ and $\mathrm{T}_{\text {ref }}$ is the temperature registered by the K-thermocouple (K) and $E_{\text {surr }}$, the energy flux emitted by the surroundings $\left(\mathrm{W} / \mathrm{m}^{2}\right)$.

Figure 1 shows the surface temperature of the sample and the emissivity calculated for A samples. It is possible to observe that until 15 min the drying temperature is not reached (remaining at wet temperature), indicating that the mobility of the water inside the Ca(II)alginate matrix is so high because the water transport is controlled by the superficial evaporation, from this minute, the internal mechanisms regulate the dehydration process. 


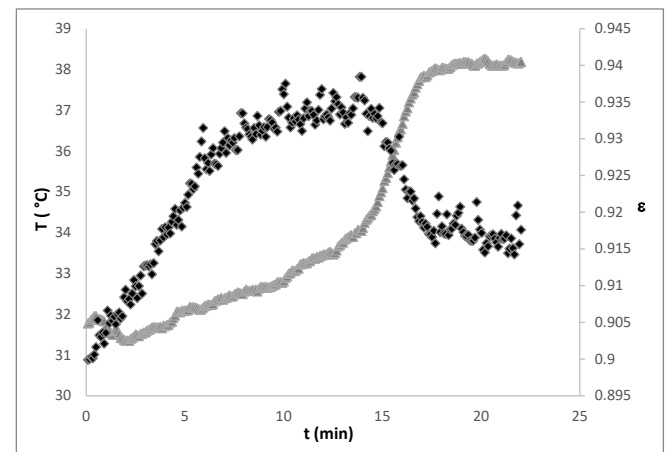

Fig. 1. Surface temperature variation ( $\diamond)$ and emissivity evolution ( $\triangle$ ) of alginate beads.

In order to model the transport, it has been necessary to characterize the mean water activity of the beads with regard to their moisture. Figure 2 shows the sorption isotherms obtained by the dynamic technique ${ }^{[8]}$, for each sample.

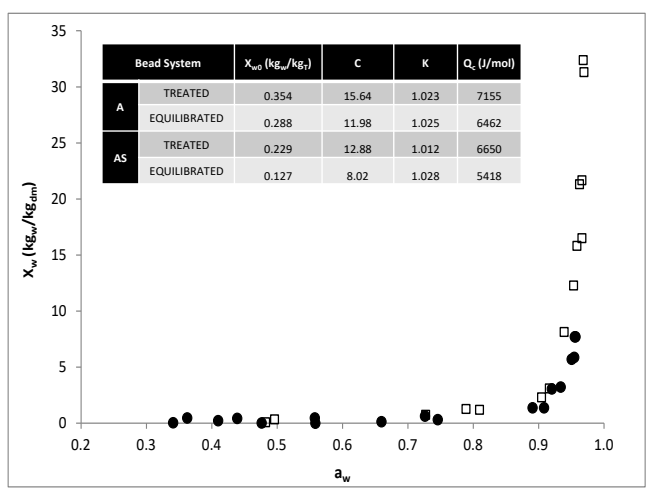

Fig. 2. Desorption isotherm (equilibrated samples), where () corresponds to alginate and (•) alginate-sucrose beads.The table shows the GAB parameters obtained.

With the aim to describe the different behaviors involved in the drying process, a thermodynamic approach has been developed. Gibbs free energy variation can be explained by the following equation ${ }^{[15]}$ :

$$
d G=-S d T+V d P+F d l+\psi d e+\sum_{i} \mu_{i} d n_{i}
$$

Where $S d T$ corresponds to the thermic term and it is directly related to heat fluxes, $V d P$ and $F d l$ are the mechanical energies related to the structural changes; corresponding to the pressure variation and to the elongation force, respectively; and $\psi d e$ represents the effect of the electric field induced by solved ions. The term $\sum_{i} \mu_{i} d n_{i}$ corresponds to the activity term and is the addition of the chemical potentials of the " $i$ " component, being constant the rest of state variables.

If the variation of free energy per mole of water is considered, it is possible to define the extended water chemical potential $\left(\Delta \mu_{w}\right)(\mathrm{J} / \mathrm{mol})$ as the quotient between the variation of the Gibbs free energy $(\mathrm{J})$ and the variation of moles of water (mol). From the extended water chemical potential and Eq. 2 it is possible to obtain Eq. 3. It should be note that the terms $F d l$ and $\psi d e$ can be neglected because $\mathrm{Ca}(\mathrm{II})$-alginate beads are an elastic system and there is no ions effect in the medium, respectively. 


$$
\Delta \mu_{w}=-s_{w}\left(T^{a i r}-T^{s a m p}\right)+v_{w}\left(P^{a i r}-P^{s a m p}\right)+R T^{s a m p} \ln \frac{a_{w}^{\text {samp }}}{\varphi^{a i r}}
$$

Where $\mathrm{s}_{\mathrm{w}}$ corresponds to the partial molar entropy of water $(\mathrm{J} / \mathrm{K} \mathrm{mol}) ; \mathrm{v}_{\mathrm{w}}$, the partial molar volume of water $\left(\mathrm{m}^{3} / \mathrm{mol}\right)$; $\mathrm{P}$, the pressure $(\mathrm{Pa})$; R, the ideal gases constant $(8.314472 \mathrm{~J} / \mathrm{K}$ mol) and $\varphi$, the relative moisture. The superscripts samp and air refer to the sample and the air surrounding it, respectively.

Once the entropic and the activities terms are obtained, it is possible to calculate the water chemical potential without considering the mechanical terms $\Delta \mu_{w}^{*}$ (Fig. 3). In the Figure, it is possible to observe how, for AS beads, the gradient decreases much earlier than A beads. This is due to the effect of higher water activity values in alginate samples, which allows them to maintain a higher value of water activity for longer.

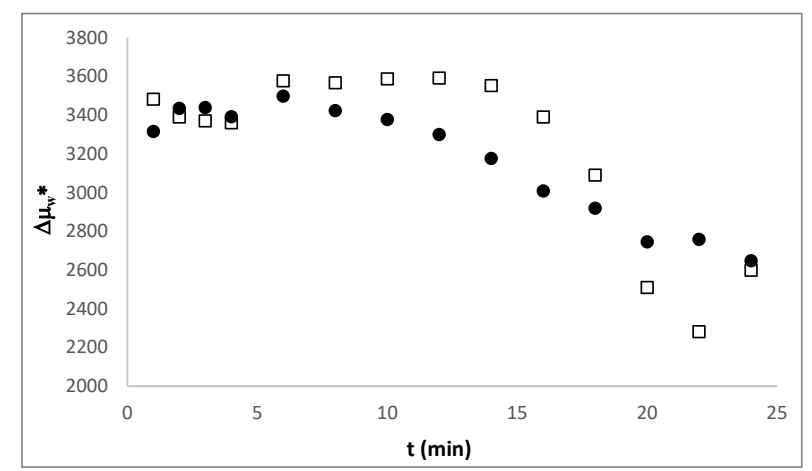

Fig. 3. Water chemical potential evolution without considering the mechanical terms, where ( $\square$ ) corresponds to alginate and (•) alginate-sucrose beads.

Applying the first relation of Onsager ${ }^{[13]}$, the water molar flux $\left(J_{w}\right)\left(\mathrm{mol} / \mathrm{s} \mathrm{m}^{2}\right)$ is related to the water chemical potential, as a driving force of the water transport, by the phenomenological coefficient $\left(L_{w}\right)\left(\mathrm{mol}^{2} / \mathrm{J} \mathrm{s} \mathrm{m}^{2}\right)$ (Equation 4).

$$
J_{w}=L_{w} \cdot \Delta \mu_{w}
$$

Applying Eq. 4 with data of chemical potential without considering the mechanical terms and the water fluxes, it is possible to obtain the phenomenological coefficient without considering the mechanical term $\left(L_{w}^{*}\right)$. Figure 4 shows the evolution of the phenomenological coefficient, calculated without mechanical terms during drying. It is possible to observe how the coefficient increases from minute 15 to compensate the imbalance between the water flux and the chemical potential obtained from the terms of activity and entropy, when it would be expected to follow a proportionality between both extensive variables as the relation of Onsager shows.

There is a linear relationship between the phenomenological coefficient without considering the mechanical terms and the water flux during the entire drying process in both systems. Therefore, following this linear prediction of the phenomenological coefficient and extending 
it for the entire drying treatment, the mechanical terms could be estimated from the following equation:

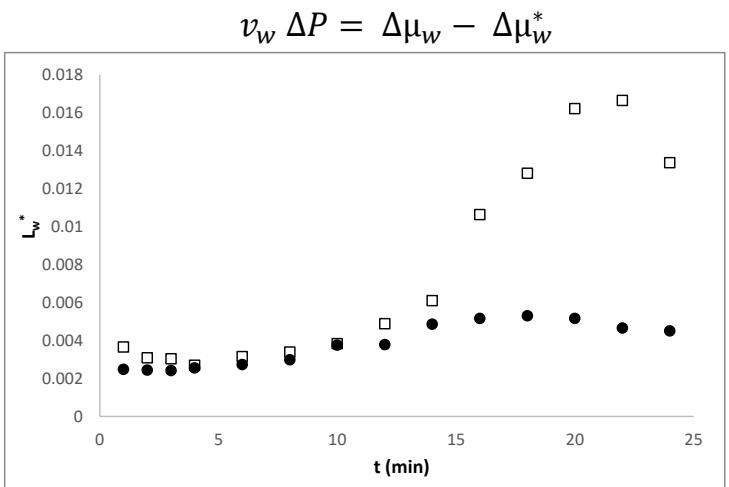

Fig. 4. Phenomenological coefficient evolution during drying, where ( $\square$ ) corresponds to alginate and (•) alginate-sucrose beads.

Figure 5 shows the evolution of the pressure term during drying process for both systems. As can be observed in this figure, the alginate samples suffer strong over atmospheric pressures from the minute 15 of the process caused by a great contraction of the system, which greatly accelerates the dehydration process. However, alginate-sucrose samples suffer the contraction process from minute 10 , generating minor overpressures. This indicates that the addition of sucrose to the $\mathrm{Ca}$ (II)-alginate structure weakens the mechanical resistance to the shrinkage of the matrix, reducing its capacity to store liquid phase.

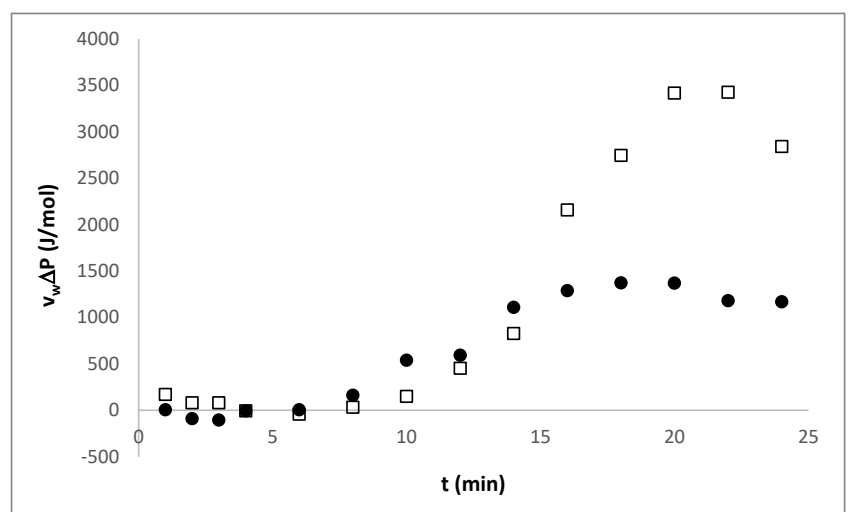

Fig. 5. Evolution of the pressure term during drying process, where ( $\square$ ) corresponds to alginate and 4. Conclusions

(•) alginate-sucrose beads.

It has been demonstrated that IRT is a good tool to control the drying process of Ca(II)alginate beads, providing valuable information about the heat transfer in colloidal systems, being possible to obtain the evolution of the beads emissivity during drying. 
It has been developed a thermodynamic model based in Gibbs free energy. The desorption isotherm was also obtained.

\section{Acknowledgements}

The authors acknowledge the financial support from the Spanish Ministerio de Economía, Industria y Competitividad, AGL2016-80643-R, Agencia Estatal de Investigación (AEI) and Fondo Europeo de Desarrollo Regional (FEDER) and Agencia Nacional de Promoción Científica y Tecnológica (ANPCyT PICT 20130434 and 2013 1331), CIN-CONICET (PDTS $2015 n^{\circ}$ 196). A special thanks to the Consejo Nacional de Investigaciones Científicas y Técnicas of Argentina for the posdoc grant of Patricio Santagapita.

\section{References}

[1] Santagapita, P. R., Mazzobre, M. F., \& Buera, M. P. (2012). Invertase stability in alginate beads: Effect of trehalose and chitosan inclusion and of drying methods. Food Research International, 47(2), 321-330.

[2] Aguirre Calvo, T. R., Busch, V. M., \& Santagapita, P. R. (2017). Stability and release of an encapsulated solventfree lycopene extract in alginate-based beads. LWT-Food Science and Technology, 77, 406-412.

[3] Traffano-Schiffo, M. V., Castro-Giraldez, M., Fito, P. J., \& Santagapita, P. R. (2017a). Encapsulation of lactase in Ca (II)-alginate beads: Effect of stabilizers and drying methods. Food Research International, 100, $296-303$.

[4] Traffano-Schiffo, M. V., Aguirre Calvo, T. R., Castro-Giraldez, M., Fito, P. J., \& Santagapita, P. R. (2017b). Alginate beads containing lactase: stability and microstructure. Biomacromolecules, 18, 1785-1792.

[5] Rahman, M. S., \& Labuza, T. P. (1999). Water activity and food preservation. In M. S. Rahman (Ed.), Handbook of food preservation. New York: Marcel Dekker, pp. 448-471.

[6] Clemente, G., Sanjuán, N., Cárcel, J. A., \& Mulet, A. (2014). Influence of temperature, air velocity, and ultrasound application on drying kinetics of grape seeds. Drying technology, 32(1), 68-76.

[7] Kumar, C., Karim, M. A., \& Joardder, M. U. (2014). Intermittent drying of food products: A critical review. Journal of Food Engineering, 121, 48-57.

[8] Traffano-Schiffo, M. V., Castro-Giraldez, M., Colom, R. J., \& Fito, P. J. (2015). Study of the application of dielectric spectroscopy to predict the water activity of meat during drying process. Journal of Food Engineering, 166, 285-290.

[9] Vadivambal, R., \& Jayas, D. S. (2011). Applications of thermal imaging in Agriculture and Food Industry-A Review. Food and Bioprocess Technology, 4, 186-199.

[10] Clemente, G., Bon, J., Sanjuán, N., Mulet, A. (2011). Drying modelling of defrosted pork meat under forced convection conditions. Meat Science, 88 (3), 374-378.

[11] Smidsrød, O., Larsen, B., Painter, T., \& Haug, A. (1969). The role of intramolecular autocatalysis in the acid hydrolysis of polysaccharides containing 1,4-linked hexuronic acid. Acta Chemica Scandinavica, 23(5), 15731580.

[12] Traffano-Schiffo, M. V., Castro-Giraldez, M., Fito, P. J., Perullini, M., \& Santagapita, P. R. (2018). Gums induced microstructure stability in Ca (II)-alginate beads containing lactase analyzed by SAXS. Carbohydrate Polymers, 179, 402-407.

[13] Traffano-Schiffo, M. V., Castro-Giráldez, M., Fito, P. J., \& Balaguer, N. (2014). Thermodynamic model of meat drying by infrarred thermography. Journal of Food Engineering, 128, 103-110.

[14] Aguirre Calvo, T., \& Santagapita, P. (2016). Physicochemical characterization of alginate beads containing sugars and biopolymers. Journal of Quality and Reliability Engineering, 2016, 1-7.

[15] Castro-Giraldez, M., Fito, P. J., \& Fito, P. (2010). Non-equilibrium thermodynamic approach to analyze the pork meat (Longissimus dorsi) salting process. Journal of Food Engineering, 99(1), 24-30. 